\title{
Percutaneous Embolization of a Hepatic Artery Pseudoaneurysm under Combined Angiographic and Fluoroscopic Guidance
}

\author{
Yama Kharoti, Khashayar Farsad \\ Dotter Interventional Institute, Portland, USA \\ Email: kharotiy@ohsu.edu
}

Received 23 April 2014; revised 25 May 2014; accepted 2 June 2014

Copyright (C) 2014 by authors and Scientific Research Publishing Inc.

This work is licensed under the Creative Commons Attribution International License (CC BY). http://creativecommons.org/licenses/by/4.0/

(c) (i) Open Access

\begin{abstract}
Hepatic artery pseudoaneurysm is a rare complication of liver directed procedures. Several strategies for treatment of pseudoaneurysms have been described. We describe a challenging case in which a small bleeding hepatic artery pseudoaneurysm is complicated by a proximal hepatic arterial occlusion, preventing transcatheter access. The aneurysm was too small to be seen on ultrasound. Using combined angiographic and fluoroscopic guidance, however, the aneurysm was triangulated and successfully treated with percutaneous direct thrombin injection. Combined angiography and fluoroscopy guided percutaneous thrombin injection of intrahepatic pseudoaneurysms can be a valuable treatment approach, particularly when other methods are not feasible or have failed.
\end{abstract}

\section{Keywords}

Pseudoaneurysm Treatment, Thrombin, Interventional Radiology, Fluoroscopy, Angiography, Hepatic Artery

\section{Case}

A 60-year-old female with a transhepatic biliary abscess drainage catheter presented with spontaneous bloody output from her catheter. The patient had a history of a remote laparoscopic cholecystectomy which was complicated by biliary duct injury and proper hepatic artery ligation. This resulted in substantial hepatic infarction requiring a left hepatectomy and hepaticojejunostomy. The patient also developed a biliary-cutaneous fistula. One month prior to presentation, a dilated and infected peripheral right hepatic duct branch was treated with a 14 Fr percutaneous drainage catheter through the fistula tract (Figure 1). It was through this catheter that the pa- 

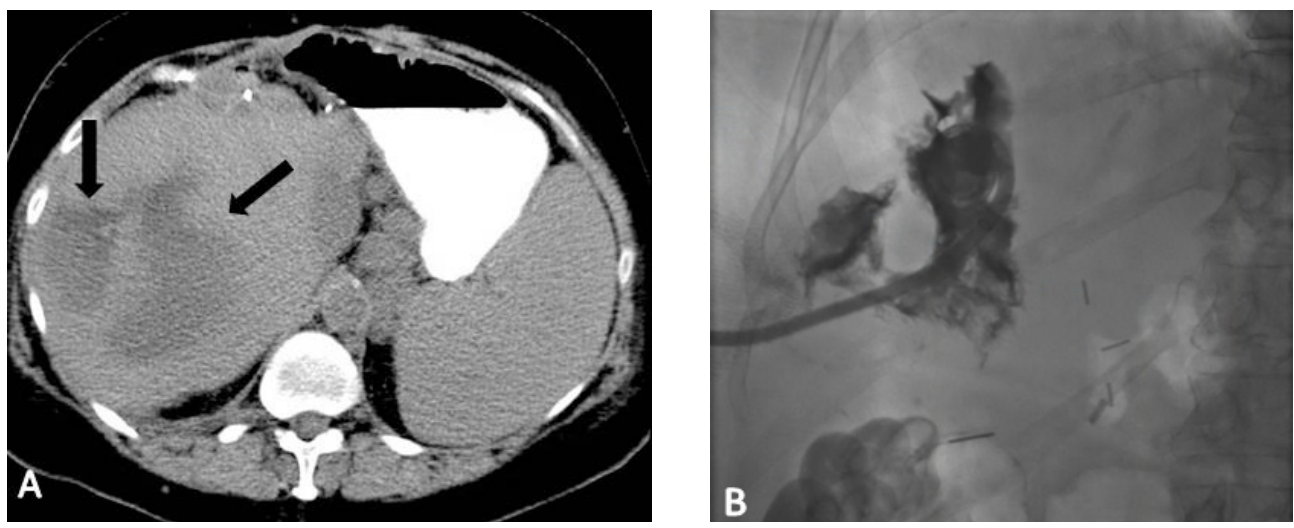

Figure 1. (A) An image from the CT done one month prior to presentation shows a large irregular hypodensity in the liver representing a hepatic abscess (arrows); (B) The abscess was treated with placement of a 14 French percutaneous drainage catheter.

tient began having pulsatile bloody discharge, and there was concern that the drain had eroded into an adjacent hepatic artery branch. On presentation, the patient was hemodynamically stable and afebrile. She had a normal platelet count and an elevated INR of 1.5 .

Review of an angiogram done at an outside facility uncovered a $6 \mathrm{~mm}$ hepatic arterial pseudoaneurysm involving a branch distal to the ligated proper hepatic artery, and filling via small collaterals. The jailed pseudoaneurysm was identified coming off of a branch from the right hepatic artery adjacent to the drainage catheter. A repeat angiogram at our institution demonstrated that the collaterals could not provide arterial access to the pseudoaneurysm (Figure 2).

At this point, the 5 French angiographic catheter and sheath were left in the patient and attached to a saline drip. The patient was taken to CT where percutaneous CT guided direct puncture of the pseudoaneurysm and thrombin injection was attempted with opacification of the pseudoaneurysm using the transarterial catheter (Figure 3). Two hundred units of thrombin were injected into the pseudoaneurysm after documentation of blood return from the needle. The patient returned to the angiography suite; however, a repeat angiogram showed continued perfusion of the pseudoaneurysm.

A second attempt was made to treat the pseudoaneurysm percutaneously under fluoroscopic guidance (Figure 4). A 20-gauge Chiba needle was triangulated into the pseudoaneurysm with intermittent opacification via the transarterial catheter. 500 units of thrombin were injected after confirmation of blood return. Repeat angiography showed no further filling of the pseudoaneurysm with preservation of distal branches. No intravasation was seen when injecting the drainage catheter. Repeat angiography with the drainage catheter withdrawn over a wire confirmed successful occlusion of the pseudoaneurysm. To prevent further erosion of the drainage catheter into the hepatic vasculature, the $14 \mathrm{Fr}$ multipurpose drainage catheter was exchanged for a smaller profile $10 \mathrm{Fr}$ Dawson Mueller drain. The patient tolerated the procedure well. Her only complication was acute kidney injury with her creatinine rising to 1.8 on post-operative day one and returning to baseline the following day. No further bleeding was seen from the biliary drain and the patient was discharged on post-operative day 4 .

\section{Discussion}

Hepatic artery pseudoaneurysm is a rare complication of liver directed procedures. Most cases occur from liver transplantation with an incidence up to 1.3\% [1]. Intrahepatic pseudoaneurysms tend to result from trauma, iatrogenic or otherwise [2] [3]. In this patient, the cause was most likely a combination of the drainage catheter placement and recent infection.

Several strategies for treatment of pseudoaneurysms have been described [4] [5]. Options for visceral pseudoaneurysm treatment include exclusion with a covered stent or embolization with coils or liquid embolic agents such as N-butyl cyanoacrylate or Onyx. Iatrogenic femoral artery pseudoaneurysms are commonly treated with ultrasound guided direct thrombin injection and have good results [6]. Cope described successful percutaneous thrombin injection into a true hepatic artery aneurysm in 1986 [2]. More recently, multiple cases have been published in which intrahepatic pseudoaneurysms were treated with percutaneous thrombin injection [3] [7] [8]. 


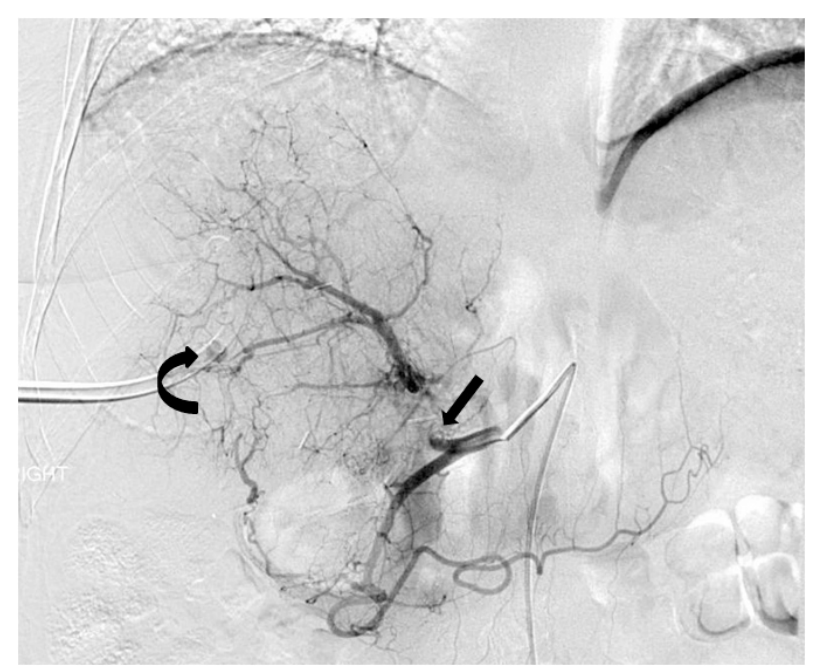

Figure 2. A visceral angiogram was done after the patient presented with bleeding from the drainage catheter. A $6 \mathrm{~mm}$ pseudoaneurysm is seen adjacent to the drainage catheter (curved arrow), arising from a branch of the right hepatic artery. The proper hepatic artery is ligated proximally (straight arrow) with distal filling from small collateral vessels.

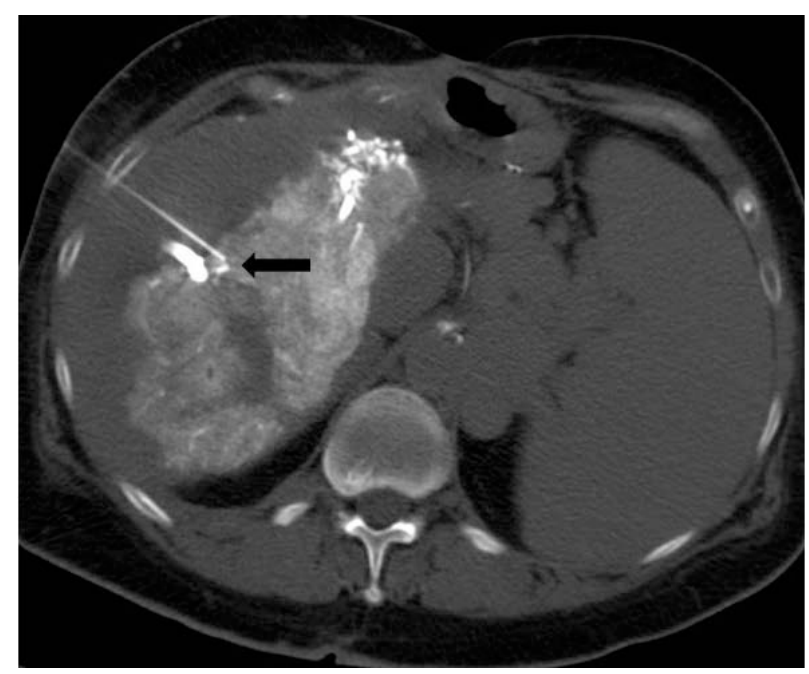

Figure 3. The 5 French angiographic catheter was left in place for the CT guided direct puncture of the pseudoaneurysm (arrow). Intermittent opacification of the pseudoaneurysm was done through the catheter to help with localization.

When the pseudoaneurysm is large and easily localized with ultrasound, one may proceed directly to percutaneous thrombin injection; however, smaller pseudoaneurysms may require an angiogram to localize for transarterial treatment. In the case presented, the ligated proper hepatic artery precluded endovascular therapy, and the pseudoaneurysm was too small to see by US, leading to the combined approach with transarterial localization and percutaneous thrombin injection.

CT and ultrasound are most commonly used to guide percutaneous thrombin injections [3] [7] [8]. We initially attempted a CT guided approach but saw persistent flow in the pseudoaneurysm on the post-procedure angiogram. Although blood return was present with the needle in the anticipated location within the pseudoaneurysm, the lesion was small enough even to make localization by CT difficult, and the needle position very well might have changed with manipulation prior to thrombin injection. With the patient on the fluoroscopy table, we were 

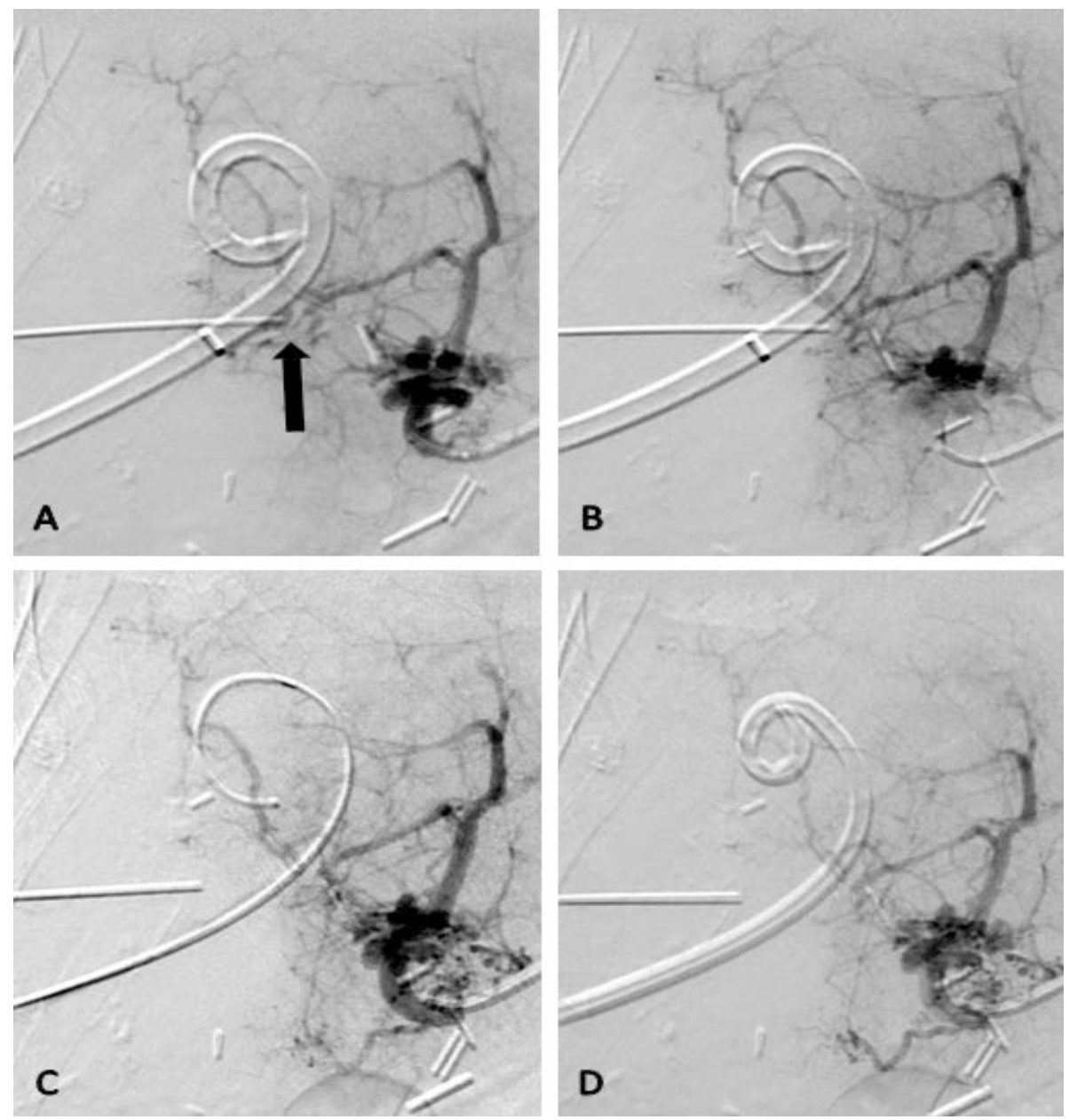

Figure 4. (A) Intermittent opacification of the pseudoaneurysm from the angiographic catheter allowed fluoroscopic triangulation and direct puncture of the pseudoaneurysm with a 20-gauge Chiba needle (arrow); (B) After thrombin injection, the pseudoaneurysm was no longer opacified with distal vessels remaining patent; (C) The drainage catheter was exchanged for a guidewire and repeat angiogram shows no filling of the pseudoaneurysm; (D) A final spot film and angiogram were done after the guidewire was exchanged for a 10 French Dawson Mueller drain.

able to triangulate the position of the pseudoaneurysm using a combination of orthogonal-view angiograms. The pseudoaneurysm was successfully injected and thrombosed with no further bleeding and with preservation of the distal branch vessels.

To our knowledge, no cases of combined angiography and fluoroscopy-guided percutaneous thrombin injection have been published. There are several advantages to this approach. The procedure is readily done on the fluoroscopy table without the need for new equipment. There is a smooth transition from angiogram to percutaneous localization and back to angiogram. Smaller lesions are better visualized on angiogram, especially when they are adjacent to drainage catheters or other equipment which may cause significant artifact on ultrasound. Lastly, confirming occlusion of the pseudoaneurysm by removing the drainage catheter over a wire and testing angiographically could be performed readily.

Combined angiographic and fluoroscopic guided percutaneous thrombin injection of intrahepatic pseudoaneurysms can be a valuable treatment approach, particularly when other methods are not feasible or have failed.

\section{References}

[1] Patel, J.V., Weston, M.J., Kessel, D.O., Prasad, R., Toogood, G.J. and Robertson, I. (2003) Hepatic Artery Pseudoa- 
neurysm after Liver Transplantation: Treatment with Percutaneous Thrombin Injection. Transplantation, 75, 17551757. http://dx.doi.org/10.1097/01.TP.0000063936.94587.10

[2] Cope, C. and Zeit, R. (1986) Coagulation of Aneurysms by Direct Percutaneous Thrombin Injection. American Journal of Roentgenology, 147, 383-387. http://dx.doi.org/10.2214/ajr.147.2.383

[3] Dambrin, C., Marcheix, B., Birsan, T., Cron, C., Muscari, F., Suc, B. and Rousseau, H. (2005) Posttraumatic Pseudoaneurysm of the Hepatic Artery: Treatment with Ultrasound-Guided Percutaneous Transhepatic Thrombin Injection. The Journal of Trauma and Acute Care Surgery, 59, 239-242. http://dx.doi.org/10.1097/01.TA.0000171526.24911.B2

[4] Reber, P.U., Baer, H.U., Patel, A.G., et al. (1998) Superselectivemicrocoil Embolization: Treatment of Choice in HighRisk Patients with Extrahepatic Pseudoaneurysms of the Hepatic Arteries. Journal of the American College of Surgeons, 186, 325-330. http://dx.doi.org/10.1016/S1072-7515(98)00032-5

[5] Yamakado, K., Nakatsuka, A., Tanaka, N., et al. (2000) Transcatheter Arterial Embolization of Ruptured Pseudoaneurysms with Coils and n-Butyl Cyanoacrylate. Journal of Vascular and Interventional Radiology, 11, 66-72. http://dx.doi.org/10.1016/S1051-0443(07)61284-6

[6] Ferguson, J.D., Whatling, P.J., Martin, J., et al. (2001) Ultrasound Guided Percutaneous Thrombin Injection of Iatrogenic Femoral Artery Pseudoaneurysms after Coronary Angiography and Intervention. Heart, 85, e5. http://dx.doi.org/10.1136/heart.85.4.e5

[7] Schuster, R., Broumandi, D.D., Lee, A.A. and Waxman, K. (2005) Percutaneous Thrombin Injection to Treat a PostTraumatic Hepatic Pseudoaneurysm. The Journal of Trauma and Acute Care Surgery, 59, 1022-1024. http://dx.doi.org/10.1097/01.ta.0000188002.05695.a3

[8] Malaisrie, S.C., Borge, M.A., Glynn, L., Santaniello, J.M., Esposito, T.J., Davis, K.A. and Luchette, F.A. (2005) Combined Percutaneous and Angiographic Thrombosis of a Traumatic Hepatic Artery Pseudoaneurysm in a Child. The Journal of Trauma and Acute Care Surgery, 59, 1025-1028. http://dx.doi.org/10.1097/01.ta.0000187970.56941.28 